\title{
Mechanisms of Practice-Related Reductions of Dual-Task Interference with Simple Tasks: Data and Theory
}

\author{
Tilo Strobach ${ }^{1}$ and Torsten Schubert ${ }^{2,3}$ \\ ' Department of Psychology, Medical School Hamburg, Germany \\ ${ }^{2}$ Institute for Psychology, Humboldt-Universität zu Berlin, Germany \\ ${ }^{3}$ Department of Psychology, Martin-Luther University Halle-Wittenberg, Germany
}

ABSTRACT

In dual-task situations, interference between two simultaneous tasks impairs performance. With practice, however, this impairment can be reduced. To identify mechanisms leading to a practicerelated improvement in sensorimotor dual tasks, the present review applied the following general hypothesis: Sources that impair dual-task performance at the beginning of practice are associated with mechanisms for the reduction of dual-task impairment at the end of practice. The following types of processes provide sources for the occurrence of this impairment: (a) capacity-limited processes within the component tasks, such as response-selection or motor response stages, and (b) cognitive control processes independent of these tasks and thus operating outside of componenttask performance. Dual-task practice studies show that, under very specific conditions, capacitylimited processes within the component tasks are automatized with practice, reducing the interference between two simultaneous tasks. Further, there is evidence that response-selection stages are shortened with practice. Thus, capacity limitations at these stages are sources for dual-task costs at the beginning of practice and are overcome with practice. However, there is no evidence demonstrating the existence of practice-related mechanisms associated with capacity-limited motor-response stages. Further, during practice, there is an acquisition of executive control skills for an improved allocation of limited attention resources to two tasks as well as some evidence supporting the assumption of improved task coordination. These latter mechanisms are associated with sources of dual-task interference operating outside of component task performance at the begin-

ning of practice and also contribute to the reduction of dual-task interference at its end.

dual tasks, task interference,

PRP, task automatization,

task coordination skills,

executive control skills

\section{INTRODUCTION}

When people execute two tasks simultaneously, performance in one or in both of the tasks is often impaired, as indicated by an increase in processing time and/or in error rates (e.g., Kahneman, 1973; Pashler, 1994a; Schubert, Fischer, \& Stelzel, 2008; Telford, 1931; Welford, 1952; for recent reviews see Fischer \& Plessow, 2015; Strobach, Schütz, \& Schubert, 2015). These performance impairments in dual-task relative to single-task situations are referred to as dual-task costs. In particular, simple, well-controllable, discrete sensorimotor tasks demonstrated these costs in dual tasks. Most of the simple tasks discussed in this paper involve a fairly straightforward stimulus-response mapping, and they usually take less than a second for someone to carry out. The relative simplicity of these tasks allows one to test precise implications of dual-task costs (in contrast to tests with more global or continuous tasks, such as car driving or writing).

In the present review, these implications are focused on the fact that a number of studies with simple tasks found that dual-task processing is optimized as a result of extended practice (e.g., Ahissar, Laiwand,

Corresponding author: Tilo Strobach, Department of Psychology, Medical School Hamburg, Am Kaiserkai 1, 20457 Hamburg, Germany. Fax: +49 (0) 40 361226 430. Phone: +49 (0) 40361226 457. E-mail: tilo.strobach@medicalschool-hamburg.de 
\& Hochstein, 2001; Liepelt, Fischer, Frensch, \& Schubert, 2011; Liepelt, Strobach, Frensch, \& Schubert, 2011; Oberauer \& Kliegl, 2004; Ruthruff, Johnston, \& Van Selst, 2001; Ruthruff, Van Selst, Johnston, \& Remington, 2006; Strobach, Frensch, \& Schubert, 2008; Strobach, Gerstorf, Maquestiaux, \& Schubert 2015; Van Selst, Ruthruff, \& Johnston, 1999). In some cases, practice leads to a strong reduction and even a complete elimination of apparent dual-task costs. For example, Schumacher et al. (2001) asked participants to perform a dual task consisting of a visual-manual task (referred to as visual task) and an auditory-verbal choice reaction task (referred to as auditory task). In the visual task, participants responded manually by pressing a key associated with the spatial position of visually presented circles. In the auditory task, participants responded by saying one, two, or three to the pitch of three different tones. Under dual-task conditions, both tasks were presented simultaneously, while there was a separated presentation under single-task conditions. Dual-task costs, which have been measured as differences between reaction time (RT) in dual-task and single-task conditions, were relatively high at the beginning of practice. However, after extended practice, these costs were eliminated, suggesting an enormous optimization of dual-task processing. In this case, dual-task performance is optimized to the level of perfect timesharing when dual-task performance is similar to the performance of the visual and auditory tasks in single-task situations (for a critical evaluation of this time sharing assumption, see Tombu \& Jolicoeur, 2004).

Given the enormous challenges in today's dynamic world, with its complex technological innovations and demands, often including the execution of more than one task, findings like those of Schumacher et al. (2001), which display large dual-task costs at the beginning of practice, followed by major reductions of costs after practice, provide essential insights from an applied perspective. From a cognitive dualtask perspective, findings on the practice-related reduction of costs naturally raise questions about the types of mechanisms that are responsible for these improvements. Therefore, the aim of the present paper is to review these mechanisms in a structured framework. This framework is structured along the following general principle: Sources that impair dual-task performance at low levels of practice (i.e., the sources of dual-task costs in an unpracticed state before practice starts) are associated with mechanisms for the reduction of the costs at the end of practice (i.e., the mechanisms that reduce the impact of sources of dual-task impairment with practice). If this same-mechanism principle is valid, then the proposed mechanisms explain how sources of the difficulty to perform two simultaneous tasks are overcome with practice. Alternatively, if this principle does not hold, sources impairing dual-task performance at low practice levels are not associated with practice-related mechanisms and thus with the practice-related reduction of dual-task costs.

This principle paves the way for the subsequent structure of the review. First, we will illustrate processes that are associated with dual-task costs at low practice levels. To foreshadow this illustration, there are interfering processes within the component tasks as well as interfering processes of executive control located outside of these tasks. Second, we will review the dual-task practice literature to assess the validity of the same-mechanism principle. This incorporates a presentation of studies on mechanisms that are associated with reduced dual-task costs after practice located within the component tasks. Further, there will be a literature review evaluating a second set of potential mechanisms that are associated with sources of dual-task costs located outside of the component tasks.

\section{SOURCES OF DUAL-TASK COSTS}

In the following section, we will illustrate processes that are sources of dual-task costs at low practice levels. This section focuses on processes within the component tasks as well as processes independent of, and thus outside of, these tasks.

\section{Sources of Dual-task Costs Within the Component Tasks}

Dual-task situations of the psychological refractory period (PRP) type are one of the most prominent situations to investigate processes that are sources of dual-task costs and are located within simple component tasks (Pashler, 1984, 1994a; Pashler \& Johnston, 1989, 1998; Schubert, 1999; Schubert et al., 2008; Telford, 1931; Welford, 1952). In these dualtask situations, two tasks are presented in close succession with various time intervals between the onsets of the first and second task stimulus (i.e., variable stimulus onset asynchronies, SOAs), and participants are instructed to respond to Task 1 first. As illustrated in Figure 1A, the performance of the second task (Task 2) typically decreases (e.g., RTs increase) with decreasing SOA and increasing task overlap. This performance decrease indicates dual-task costs and thus dual-task interference in the context of PRP dual tasks (i.e., the PRP effect).

To explain the PRP effect, the central bottleneck theory holds that the selection of which response to execute cannot be made for two tasks in parallel. Instead, this model assumes sequential response selection of two tasks in a dual-task situation due to a structural and unavoidable processing bottleneck (see Figure 1B). This sequential processing at the central bottleneck leads to long interruptions of Task 2 processing at short versus long SOAs and, hence, the PRP effect (Pashler, 1994a). In contrast to the bottleneck at the response-selection stage, the initial perception stage (at which stimulus information is processed) and the final motor-response stage (at which there is the execution of a motor response) can run in parallel.

The central bottleneck theory makes a number of predictions that were tested empirically and supported this theory. Perhaps its most striking predictions arise when difficulties of particular processing stages of Task 2 are manipulated. Suppose that, by means of some difficulty manipulation, there is an increase of the processing duration of Task 2 stages at or after the bottleneck by $x$ ms; such a candidate stage is the response-selection stage. What does the central bottleneck theory predict? As the difficulty manipulation occurs at or after the central bottleneck stage, the consequence is that RT2 increases by the same amount of $x$ ms by which the difficulty manipulation increases, regardless of SOA. In fact, the effect of the difficulty manipulation will be additive with the PRP effect (i.e., the effect of SOA on RT2). Findings 
Panel A.

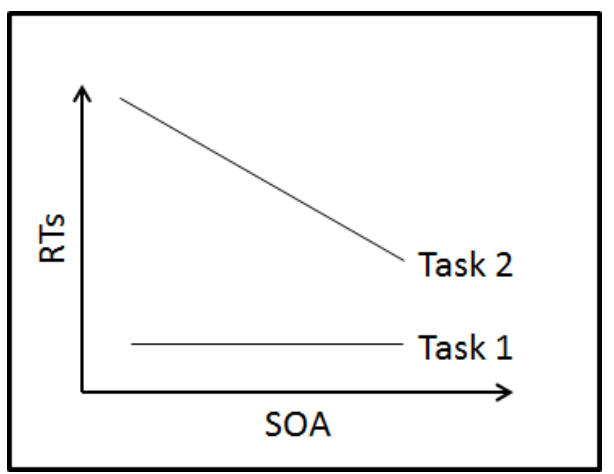

Panel B.

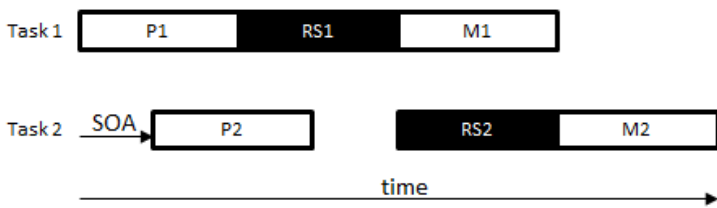

FIGURE 1.

Panel A: Illustration of typical performance pattern (i.e., reaction times [RTs]) in the context of dual tasks of the psychological refractory period (PRP) type. While performance in Task 1 is independent of the stimulus onset asynchrony (SOA), Task 2 performance is impaired with decreasing SOA. The RT increase from long to short SOAs reflects the PRP effect. Panel B: Dual-task processing architecture according to the central bottleneck model (e.g., Pashler, 1994a) in PRP dual tasks with SOA manipulations. Central response-selection (RS1; RS2) stages in Task 1 and Task 2 are processed sequentially while perception (P1; P2) and response (R1; R2) stages are processed in parallel. Response selection stages (e.g., in Task 2) are lengthened due to the manipulation of the compatibility between stimuli and responses (McCann \& Johnston, 1992) while perception stages are lengthened due to the manipulation of stimulus intensity (Pashler \& Johnston, 1989). In particular, extra time needed for the lengthened perception stage in Task 2 can run in parallel to RS1 and is thereby absorbed into the waiting time of RS2 until RS1 is completed.

of McCann and Johnston (1992) were consistent with this "additivity" prediction: When stimulus-response compatibility (assumed to be processed at the central response-selection stage and thus at the bottleneck stage) was manipulated, the compatibility effects were additive. Compatibility was varied by whether Task 2 stimuli were mapped onto their responses by rules with dimensional stimulus-response overlap or arbitrarily (symbolic arrows and spatially congruent responses or arbitrary letters, respectively). In contrast, difficulty manipulations before the bottleneck stage in Task 2 should lead to underadditive effects with SOA (i.e., similar RTs at shorter SOAs and RT differences at longer SOAs) because, at shorter SOAs, the difficulty manipulation is absorbed into slack time (i.e., the waiting time to start Task 2 response selection until Task 1 selection is completed). So, total RTs of different pre-bottleneck manipulation conditions are similar at these SOA levels. However, at longer SOAs, effects of pre-bottleneck manipulations should be obvious in RTs of Task 2 at long SOAs. In fact, when altering the intensity of Task 2 stimuli, which should affect the perception stage, the effect on RT2 was increased from $5 \mathrm{~ms}$ at short to $30 \mathrm{~ms}$ at long SOAs (Pashler \& Johnston, 1989). Thus, these and other empirical evidences supported predictions of the central bottleneck theory (Pashler \& Johnston, 1998).

Other theories accompany the central bottleneck locus by assumptions about sequential processing at peripheral stages, such as motorresponse execution when similar motor systems are required in two tasks (e.g., Bratzke, Rolke, \& Ulrich, 2009; De Jong, 1993; Sigman \& Dehaene, 2006; Sommer, Leuthold, \& Schubert, 2001; Van Selst et al., 1999). In fact, these theories assume that there is a motor responseinitiation bottleneck, preventing two responses from being initiated simultaneously or in close succession. One prediction of these theories is that a difficulty manipulation at the motor stage of Task 1 propagates into Task 2 processing. Consistent with these theories' prediction, when participants perform ballistic manual movements of different distances and processing durations in Task 1 and a manual choice RT task in Task 2, there was a propagation effect of the movement distance from RT1 on RT2 (Bratzke et al., 2009).

In sum, dual-task costs arise from bottleneck stages within the component tasks. Next to a central bottleneck stage (e.g., Pashler, 1994a), there are assumptions of peripheral bottlenecks, such as motor-stage interference (e.g., Bratzke et al., 2009). Following the same-mechanism principle, we assume that such bottleneck processes within the component tasks (i.e., at central response selection and/or motor stages) are associated with mechanisms for the reduction of dual-task interference at the end of practice-this principle is referred to as the optimized task processing principle. These practice-related mechanisms should explain how, as a result of practice, the impact of bottleneck stages on the difficulty to perform two simultaneous tasks is overcome.

\section{Sources of Dual-task Costs Outside the Component Tasks}

Alternative to the central bottleneck theory, resource theories assume that two response selections can be processed in parallel in dual tasks, but that sharing the same limited attentional resource(s) causes dualtask costs (e.g., Pashler, 1994b; Tombu \& Jolicoeur, 2003; Wickens, 2008). Consistent with this view, following instructions, participants strategically prioritize one task over another (e.g., Gopher, Brickner, \& Navon, 1982; Navon \& Gopher, 1979; Norman \& Bobrow, 1975). This strategic prioritization is particularly evident in situations with the freedom to prioritize and to allocate attention to different tasks; the latter rather requires complex and/or self-paced tasks (Bier, de Boysson, \& Belleville, 2014; Korteling, 1991). Next to this phenomenon of task prioritization, resource theories were supported by interpretation of invariances of RT1 to SOA and Task 2 difficulty manipulations (Navon \& Miller, 2002). Recent representatives of resource theories (e.g., Logan \& Gordon, 2001; Meyer \& Kieras, 1997; Navon \& Miller, 2002; Tombu 
\& Jolicoeur, 2003) assume that sequential processing, as anticipated in the central bottleneck theory, may be a strategic by-product of flexible scheduling of limited attentional resources (e.g., attention allocation of $100 \%$ to Task 1 and attention allocation of $0 \%$ to Task 2). However, consistent with this central bottleneck theory, strategic task prioritization does not require shared processing of two response-selection processes, but might schedule central bottleneck stages according to the total time needed for the performance of two tasks (Miller, Ulrich, \& Rolke, 2009). Irrespective of the theoretical model (central bottleneck theory, resource theory), optimization in dual-task attention allocation may thus represent a further mechanism of practice-related reduction of dual-task costs (optimized attention allocation principle) according to the same-mechanism principle. That is, this mechanism should broadly explain how scheduling of limited attentional resources as a source of the difficulty to perform two simultaneous tasks is overcome with practice.

Recent studies applied variations of the PRP paradigm for analyses of executive control functions beyond attention allocation (Jiang, Saxe, \& Kanwisher, 2004; Kamienkowski, Pashler, Sigman, \& Dehaene, 2011; Liepelt, Strobach, et al., 2011). Such executive functions are generalpurpose control mechanisms that regulate the dynamics of human cognition and action (Miyake et al., 2000; Miyake \& Friedman, 2012) and, among others, coordinate the processing of two task streams of a PRP dual task (e.g., De Jong, 1995; Luria \& Meiran, 2003; Schubert \& Szameitat, 2003; Sigman \& Dehaene, 2006; Szameitat, Lepsien, von Cramon, Sterr, \& Schubert, 2006). For example, when participants are faced with dual-task trials compared to single-task trials, one can observe a general increase of RTs for Task 1 in PRP dual tasks compared to single-task RTs, which points to the action of time-consuming coordination processes at the beginning of dual-task trials (e.g., Jiang et al., 2004). From a perspective on executive processes, dual-task performance data may thus point to a set of well-identifiable task coordination processes. According to the same-mechanism principle, we assume that such processes are associated with mechanisms for the reduction of dual-task costs at the end of practice. These practice-related mechanisms should explain how the impact of task coordination processes on the difficulty to perform two simultaneous tasks is overcome due to the acquisition of task coordination skills (optimized task coordination principle).

\section{MECHANISMS OF PRACTICE-RELATED OPTIMIZATION OF DUAL-TASK PERFORM- ANCE}

This section represents the "heart" of this review. That is, it evaluates the validity of the same-mechanism principle, assuming that sources that impair dual-task performance and add to dual-task costs at low levels of practice are associated with mechanisms for the costs' reduction at the end of practice. The same-mechanism principle is evaluated with respect to the task processing optimization principle (i.e., the mechanisms reducing interference of processes located within the component tasks) as well as the optimized attention allocation and optimized task coordination principles (i.e., the mechanisms reducing interference of processes located outside of the component tasks).

\section{Mechanisms Within the Component Tasks}

The dual-task practice literature investigates the optimized task processing principle and thus the impact of skills for optimized component task processing on improved dual-task performance from two perspectives. First, which specific processing stages within simple component tasks are optimized and thus shortened as a result of dual-task practice: the initial stimulus perception stage, the central response-selection stage, the final motor stage, or a combination of these stages (Pashler \& Baylis, 1991)? Although there is agreement in the literature assuming that these stages are not truly successive and have a fairly high degree of interactive influence (e.g., Levy \& Pashler, 1995; Pashler, 1994a), the distinction of perception, response-selection, and motor stages provides a systematic framework to structure the literature on stage shortening. Second, are component-task skills able to lead to task automatization and thus to the elimination of within-task capacity limitations (i.e., processing bottlenecks) and dual-task costs? In the following two paragraphs, both questions are elaborated separately.

\section{WHICH SPECIFIC PROCESSING STAGES WITHIN THE COMPONENT TASKS ARE SHORTENED AS A RESULT OF DUAL-TASK PRACTICE?}

Some practice studies have already addressed the question of the locus of practice effects within simple tasks in dual tasks of the PRP type (e.g., Ahissar et al., 2001; Anderson, Taatgen, \& Byrne, 2005; Dux et al., 2009; Garner, Tombu, \& Dux, 2014; Kamienkowski et al., 2011; Ruthruff et al., 2001, 2006; Sangals, Wilwer, \& Sommer, 2007; Strobach, Liepelt, Pashler, Frensch, \& Schubert, 2013; Van Selst et al., 1999). For example, Ruthruff et al. (2006) addressed this question by comparing the performance of three groups of participants under different learning conditions: While Group 1 practiced an auditory-verbal (Task 1) and a visual-manual (Task 2) task in a PRP dual-task situation for eight sessions, Group 2 practiced only Task 1, and Group 3 practiced only Task 2 for the same amount of time. In the 9th session, all three groups performed the same dual task as Group 1 performed during practice. One of the main findings of Ruthruff et al. (2006) was a similar reduction of the dual-task costs in Task 2 (i.e., the PRP effect) in the dualtask learning Group 1 as well as in Group 2 that practiced only Task 1. (Group 3 showed still increased dual-task costs.) In addition, the authors found that the practice-related shortening of the processing time in Task 1 was closely related to the practice-related reduction of these costs. Presuming a processing limitation at the central response selection stage, the authors interpreted these findings with the assumption that the practice-related optimization of dual-task processing is related to the reduction of the processing time of the pre-motor stages in the component tasks.

However, these findings provided no conclusive evidence about the exact location of practice-related stage shortening within task processing streams. In fact, the findings of Ruthruff et al. (2006) do 
not clarify which of the pre-motor stages have been shortened; the speed-up could occur at the perception stage, the response-selection stage, or a combination of both stages. The same is true for studies that investigated the electroencephalographic (EEG) lateralized readiness potential in the context of dual-task learning, providing neuronal evidence for practice-related shortenings at pre-motor task stages (e.g., Sangals et al., 2007).

First studies aiming to precisely locate stage shortening in component tasks made use of the phenomenon of the backward compatibility effect. This effect is demonstrated by RT1 that is shorter when Task 2 requires a compatible versus an incompatible response to the response in Task 1 (e.g., say left and press the left key vs. say left and press the right key, e.g., Ellenbogen \& Meiran, 2008; Hommel, 1998; Hommel \& Eglau, 2002; Watter \& Logan, 2006). This phenomenon may be due to an overlap and parallel processing of some portions of stimulusresponse mappings in both tasks (e.g., Hommel, 1998; Janczyk, Pfister, Hommel, \& Kunde, 2014). In a practice study, Thomson, Danis, and Watter (2015) showed that the size of the backward compatibility effect is closely associated with the duration of the response-selection stage in Task 1. When this stage was shortened through PRP practice with a letter (Task 1) and a color discrimination task (Task 2) across six sessions, the magnitude of the effect decreased. In turn, when the duration of Task 1 response selection was increased after the end of practice by increasing the number of stimulus-response mapping rules (by increasing from a two-letter discrimination task during practice to a four-letter discrimination task after the end of practice), the backward compatibility effect increased by a magnitude similar to this increased duration. These findings are consistent with the assumption that the response selection stage is shortened with practice and this shortening might contribute to the practice-related reduction of dual-task costs. However, identification of the shortened stage by analyzing the modulation in the backward compatibility effect is not completely unequivocal. First, there are findings that do not limit this effect to interference at Task 1's response-selection stage but also locate this interference within alternative processes, such as stimulus evaluation (e.g., Masaki, Takasawa, \& Yamazaki, 2000) or response activation (e.g., Hommel, 1998). Thus, the reduction of the backward compatibility effect can result from shortenings other than response selection. Second, identification of the shortened stage via the reduction of the backward compatibility effect is, by its definition, limited to response selection. That is, this approach does not allow for systematic investigation of shortenings at initial perception and/or final motor stages. As a consequence, it does not allow investigating the optimized task processing principle in the context of a motor stage bottleneck (e.g., Bratzke et al., 2009) and associated mechanisms for improved dual-task performance after practice. Third, stage shortenings by means of the modulation of the backward compatibility effect are limited to shortenings in a first or shorter component task and the generalizability of this analysis to a second or longer task is not viable.

To overcome these limitations, Strobach et al. (2013) applied a transfer logic originally proposed by Pashler and Baylis (1991). To identify the particular processing stages which potentially undergo a practice-related shortening at the end of practice, the authors introduced transfer manipulations separately targeting processing routines at the perception, the response-selection, and the motor stages. As a consequence, the processing routine may or may not be applied in the transfer situation. According to this transfer logic, the transfer manipulation was supposed to lead to an increase in processing time if participants cannot use a processing routine any longer that was speeded-up due to learning. On the other hand, no increase in processing time is expected if learning has not led to a speed-up of a processing routine.

Consistent across a visual and an auditory task (e.g., Schumacher et al., 2001), possible shortenings of the processing time at the responseselection stages of these tasks were assessed by comparing the performance of participants before and after a manipulation of the stimulusresponse mapping rules at the end of extensive practice. Changing the mapping rules from the end of practice to transfer resulted in a significant RT increase from the former to the latter conditions. This result suggested a practice-related shortening of stimulus-response mapping and was conclusive evidence for response-selection stage shortening. Consistent with the applied transfer logic, visual and auditory stimulus information processing was also manipulated separately. However, none of these manipulations resulted in strong RT prolongations; thus, pre-motor stage shortening during dual-task practice (Ruthruff et al., 2006) is mainly focused on the central response-selection stages (see also Garner, Lynch, \& Dux, 2016, with consistent findings in an alternative theoretical framework). Consistent with this assumption, neuroimaging data showed that improved dual-task performance after practice is associated with improved efficiency of stimulus-response processing, indicated by shorter neural activity (Dux et al., 2009) and better segregation of individual task representations (Garner \& Dux, 2015) in the prefrontal cortex.

In sum, central stage shortening in simple tasks might thus contribute to the reduction of dual-task costs and the improvement of dual-task performance with practice in the context of the optimized task processing principle; in contrast, manipulations at the perception stages do not produce evidence for shortenings of perceptual processes. The result pattern after response-selection stage manipulations is consistent with the same-mechanism principle. This stage is associated with dual-task interference at the beginning of practice and is associated with mechanisms that contribute to the reduction of this interference after practice. These conclusions were derived from dual tasks with constant and simultaneous task presentations but are consistent with findings in single tasks (Pashler \& Baylis, 1991) and assumptions in the context of PRP dual tasks (e.g., Ruthruff et al., 2006). Therefore, we assume that central stage shortening (and no perceptual stage shortening) is a generalizable phenomenon.

This particular combination of central response-selection stage shortening and the absence of perception stage shortening can result in a specific pattern (i.e., timing) of processing stages in the two tasks in which no overt (or very reduced) dual-task costs are present despite the fact that within-task capacity-limited processes are still existent. One example of such a task processing architecture is specified in the latent bottleneck model (e.g., Anderson et al., 2005; Ruthruff, Johnston, Van 
Selst, Whitsell, \& Remington, 2003; Schubert, 2008). According to this model, practice will eliminate dual-task costs if it leads to scheduling tasks in a way that the capacity-limited stage of one task finishes before the capacity-limited stage of the other begins.

Further, manual and verbal motor response information was separately manipulated in the dual-task situation of Schumacher et al. (2001; Strobach et al., 2013). However, none of these manipulations resulted in significant RT prolongations. Thus, there is no evidence for motor stage shortening during dual-task practice in this dual-task situation using different motor output modalities. Hence, in this data set, there is no evidence for a contribution of an optimized motor stage bottleneck to reduced dual-task costs after practice. However, this conclusion might result from the specific combination of different motor modalities in the component tasks (avoiding a motor bottleneck) and could differ in practice situations with responses in the same modality (e.g., two manual response tasks, see Sommer et al., 2001). This practice situation with a modality-specific motor bottleneck was tested by Ruthruff et al. (2001, 2006; see also Maquestiaux, Hartley, \& Bertsch, 2004; Van Selst et al., 1999), observing equivalent practice-related reductions of the PRP effect as well as for RT1. These equivalent reductions are consistent with the assumption that there is no shortening of processes after the central bottleneck and thus no optimized motor stage bottleneck in simple tasks. This conclusion reveals the invalidity of the same-mechanism principle in this context: The impact of a bottleneck at the final motor stages is not reduced with practice and is thus not associated with the reduction of dual-task interference. Hence, there is no evidence that all cost sources (e.g., the motor bottleneck) are associated with practice-related mechanisms for improved dual-task performance in the context of the optimized task processing principle. It therefore seems like evidence for this principle is exclusively valid for mechanisms associated with the central response-selection bottleneck.

\section{ARE COMPONENT TASK SKILLS ABLE TO LEAD TO TASK AUTOMATIZATION AND THUS TO THE ELIMINATION OF CAPACITY LIMITATIONS?}

If tasks are automatized, they should not require cognitive control and are supposed to be processed in parallel (without interference) with concurrent tasks because they do not require processing capacity (e.g., Hampson, 1989; Logan, 1988; Schneider \& Shiffrin, 1977). As a consequence, practice-related task automatization is a promising mechanism to explain the elimination of dual-task interference with practice. A series of studies (e.g., Maquestiaux, Didierjean, Ruthruff, Chauvel, \& Hartley, 2013; Maquestiaux, Laguë-Beauvais, Ruthruff, \& Bherer, 2008; Maquestiaux, Laguë-Beauvais, Ruthruff, Hartley, \& Bherer, 2010; Ruthruff et al., 2006) tested the ability of task automatization by providing extensive single-task practice with an auditory-verbal task (e.g., low and high pitched tones were mapped on verbal low and high responses, respectively) in single-task trials across multiple practice sessions. In a following test session, the practiced auditory-verbal task was combined with a new visual-manual task (e.g., the digits 1,2, 3, 4 or the letters $A, B, C, D$ were mapped on manual finger responses) in a PRP dual-task situation. In this particular PRP situation, the first visual-manual task (Task 1) preceded the auditory-verbal task (Task 2) by varying SOAs. To explain a potential PRP effect, the central bottleneck theory assumes that there is a strict sequential response selection in two tasks of a dual-task situation due to a structural and unavoidable attentional bottleneck. Such a processing bottleneck exists when task performance is not automatized.

Under the following conditions, the large majority of participants produced data consistent with this bottleneck theory: (a) single-task practice with the auditory-verbal task of $\sim 4,500$ trials and (b) four tone pitches in this task (the lower two tones were mapped on the low response while the higher two tones were mapped on the high response). In fact, 14 out of 18 participants showed clear PRP effects in a final test session when this auditory-verbal Task 2 was combined with the visual-manual Task 1 (Ruthruff et al., 2006). However, under conditions that are more favorable for task automatization, many participants produced no PRP effect. That is, after more than 5,000 single-task practice trials with the auditory-verbal task and only two tone pitches, RTs in Task 2 were rather independent of SOA (Maquestiaux et al., 2008). The authors thus concluded an ability to automatize component task processing under very specific conditions with sufficient and appropriate practice.

The conclusion of task automatization was consistent with data in an alternative dual-task test context (Hazeltine, Teague, \& Ivry, 2002; Schumacher et al., 2001). In this test context with a simultaneous presentation of an easy visual and auditory task, some participants showed no dual-task costs at the end of practice (however, see Anderson et al., 2005, modelling this elimination of dual-task costs still including within-task capacity-limited processing stages). This phenomenon of eliminated dual-task costs might occur due to stimulus and response features represented in common domains (e.g., a left circle requires a left key press response), avoiding any crosstalk between tasks. These common domains might automatize bindings between these features (Göthe, Oberauer, \& Kliegl, 2016). However, it seems rather difficult to imagine that task automatization is possible in more complex and more difficult everyday-life tasks, such as car driving and other tasks. Therefore, a contribution of task automatization to the reduction of dual-task costs with practice might be more an exception than the rule.

Nevertheless, the findings on task automatization are consistent with the assumption that there is evidence that within-task capacity limitations, as sources for the difficulty to perform two simultaneous tasks, can be overcome by practice. Accordingly, these limitations that impair dual-task performance at low levels of practice are associated with mechanisms for the reduction of the costs at the end of practice, summarized in the optimized task processing principle.

\section{Mechanisms Outside the Component Tasks}

After reviewing the impact of stage shortening and automatization of component tasks on the reduction of dual-task costs after practice (the evaluation of the optimized task processing principle), we will review a second set of mechanisms underlying this reduction of dual-task costs. 
This set of mechanisms is located outside the component tasks and is related to the optimization of executive functions (Damos \& Wickens, 1980; Hirst, Spelke, Reaves, Caharack, \& Neisser, 1980; Kramer, Larish, \& Strayer, 1995). It is represented by the optimized attention allocation and optimized task coordination principles.

The former principle (i.e., the optimized attention allocation principle) is associated with the efficiency in the strategic allocation and deployment of control of two tasks to limited processing capacities, such as the working memory component (e.g., Bier et al., 2014). With practice, participants might acquire improved attention allocation skills, leading to an optimization in dual-task attention allocation (Kramer et al., 1995) and management of task priorities through self-regulation of attention priorities (Bier et al., 2014). Exemplarily, the latter management component might train participants to strategically reduce the dual-task processing time by the optimized processing account of the total time needed for the performance of two tasks (Miller et al., 2009). Alternatively, the executive control of visual attention (ECTVA) theory (Logan \& Gordon, 2001) proposes a set of executive control parameters that determine the strategy and thus the attentional resources and breadth dedicated to the component tasks in a dual-task context. These parameters might specify attention allocation on two tasks in a way that reduces dual-task costs.

The latter principle (i.e., the optimized task coordination principle) should explain how the (interfering) impact of executive functions on the difficulty to perform two simultaneous tasks is overcome due to the acquisition of task coordination skills, contributing to the practicerelated reduction of dual-task costs (Damos \& Wickens, 1980; Liepelt, Strobach, et al., 2011). From the present perspective, such task coordination skills not only refer to broadly expressed attention allocation strategies but to well-identifiable processing components. We will come back to such specific components in latter sections.

Both the optimized attention allocation and optimized task coordination principles share the following characteristics of optimized executive control skills. First, executive control skills should not be acquired under single-task practice conditions. That is, these skills are not attributable to learning the component tasks exclusively (Damos \& Wickens, 1980; Hirst et al., 1980; Kramer et al., 1995; Logan \& Gordon, 2001; Oberauer \& Kliegl, 2004). Second, once acquired, improved skills should at least be partially independent of the specific properties of the component tasks presented during dual-task practice. Consequently, these skills should be transferable across different dual-task situations (Bherer et al., 2006; Liepelt, Strobach, et al., 2011). The subsequent sections will evaluate the literature on these characteristics of optimized skills in the context of the (a) optimized attention allocation and (b) optimized task coordination principles.

\section{THE OPTIMIZED ATTENTION ALLOCATION PRINCIPLE}

The optimized attention allocation principle proposes the acquisition of skills for an efficient strategic allocation of limited processing resources to optimally process two simultaneous tasks. Most importantly, we assume that such general skills are acquired during practice in which participants are instructed to flexibly vary their attentional resources between the two tasks by constantly varying their task response priorities (this practice type is referred to as the variable-priority condition, e.g., Kramer et al., 1995). That is, the acquisition of attention allocation skills requires the freedom to allocate attention to different tasks. Further, the freedom to allocate attention depends on the type of tasks the participants are asked to combine: While complex tasks should be beneficial for this type of skill acquisition (Bier et al., 2014), discrete tasks and tasks presented at a fixed interval should be less beneficial (e.g., Bherer et al., 2005). In contrast to a variable-priority condition, participants were instructed to emphasize both tasks constantly and equally in a fixed-priority condition. Under this condition, there should be no variation of attentional resources during practice and, therefore, no acquisition of skills for optimized attention allocation.

In a series of studies, dual-task improvement was analyzed as an effect of practice under the variable-priority and fixed-priority condition to test the acquisition of optimized attention allocation skills. For instance, participants practiced a visual detection and an alphanumeric equation task in dual tasks with a variable or a fixed-priority instruction (Bier et al., 2014; Gagnon \& Belleville, 2012; Lee et al., 2012; Voss et al., 2012; see also Bherer et al., 2005, 2006, 2008; Kramer et al., 1995; Kramer, Larish, Weber, \& Bardell, 1999). These studies assumed that if there is an acquisition of attention allocation skills, the former dualtask practice type should lead to a better dual-task performance at a final test (better dual-task performance was conceptualized as reduced dual-task costs between dual- and single-task performance). In fact, the assessment of dual-task performance with the practiced component task set indicated a larger practice-related reduction of dual-task costs under the variable-priority condition than under the fixedpriority condition. Findings of reduced dual-task costs with variablepriority practice versus fixed-priority practice are consistent with the assumption that, with variable-priority practice, there is an acquisition of skills for an efficient allocation of limited attentional resources to the processing of two simultaneous tasks. These findings are thus consistent with the assumption that optimized attention allocation contributes to the reduction of dual-task costs and to the improvement of dual-task performance with practice. Since the improved dual-task performance after variable-priority practice, in contrast to fixed-priority practice, was also produced in non-practiced transfer tasks (e.g., alphanumeric equation with letters different from the practice letters), there is evidence that skills for an efficient allocation of limited attentional resources are not specific for the practiced task characteristics but are transferable to new tasks. So, there is empirical evidence for the optimized attention allocation principle, stating that the impairment in dual-task performance as a consequence of the inefficiency in flexible scheduling of limited attentional resources at the beginning of practice is reduced. This reduction is realized by the acquisition of improved attention allocation skills.

Yet, based on the practice protocols mentioned above, there are no explicit conclusions available whether participants optimized a single strategy (e.g., total time needed for the performance of two tasks; Miller et al., 2009) or whether they qualitatively changed or adapted to a new strategy during variable-priority dual-task practice. In an 
analogy, there are, however, other dual-task practice studies that rather suggest the assumption of optimizing a single strategy. For example, when participants practice two component tasks with equal priority, dual-task costs are extremely reduced with practice, while such practice with priority on one task demonstrates significant dual-task costs from the beginning to the end of practice (Schumacher et al., 2001). In particular, the latter finding on practice with priority on one task in comparison to equal-priority practice shows that experimenter-aimed configurations and instructions have a significant influence on dualtask performance during practice. Further, these latter findings suggest potentially similar performance patterns and thus a single strategy from the beginning to the end of practice. Since this assumption stems from dual tasks with variable-priority instructions and is thus rather speculative, future studies should further track processing strategies and their potential chances in the context of variable dual-task practice with simple sensorimotor tasks (see Strobach, Schubert, Pashler, \& Rickard, 2014, for tracking strategies during dual-memory retrieval practice).

\section{THE OPTIMIZED TASK COORDINATION PRINCIPLE}

With the exception of Bier et al. (2014), none of the studies discussed so far in the context of optimized executive control skills included a control procedure with single-task practice and a comparison of dual-task performance after this practice type with dual-task performance after dual-task practice with fixed priorities. Conversely, there is no inclusion of such a fixed dual-task practice procedure if studies realized single-task practice (Garner et al., 2014). This latter, single-task practice procedure is, however, essential for investigating the acquisition of executive control skills. So, these studies allowed no investigation of well-identifiable executive processes and thus a direct test of the acquisition of task coordination skills (Damos \& Wickens, 1980; Kramer et al., 1995; Oberauer \& Kliegl, 2004). Further, from our perspective, variable-priority dual-task practice creates no practice condition that is well-controllable and allows the deduction of assumptions about well-identifiable executive processes. This is because dual-task situations during variable-priority practice typically present complex and self-paced component tasks under conditions that offer the freedom to strategically and flexibly allocate attention. Hence, such situations may not be appropriate to pinpoint well-identifiable executive control skills next to the broadly defined attention allocation skills. In the following section, we will, therefore, evaluate studies with a comparison of dual-task performance with discrete tasks after single and fixed-priority dual-task practice.

Such a comparison was conducted in Liepelt, Strobach, et al. (2011) and Strobach, Frensch, Soutschek, and Schubert (2012; see also Anguera et al., 2013; Bier et al., 2014; Lussier, Gagnon, \& Bherer, 2012). In these studies, the authors compared the dual-task performance of two groups of participants experiencing different types of practice with a visual and auditory sensorimotor task (e.g., Schumacher et al., 2001). While (a) fixed-priority dual-task practice included intermixed presentation of both tasks in dual tasks (including an SOA of $0 \mathrm{~ms}$ as well as a constant equal task priority) and in single tasks in dual-task blocks, and separate presentation of both tasks in single-task blocks, respectively (see also Hazeltine et al., 2002; Schumacher et al., 2001), (b) pure single-task practice included the exclusive presentation of the visual and auditory tasks in separate single-task blocks. In fact, after six sessions of dual-task practice, dual-task performance in the 7th test session was improved when compared to the dual-task performance after six sessions of single-task practice. In detail, this improvement was exclusively demonstrated by reduced dual-task RTs in the auditory task, while there was no such dual-task evidence in the visual task. The auditory task and the visual task are typically performed slower and faster, respectively, indicated by longer and shorter RTs in single and dual tasks (see also Hartley, Maquestiaux, \& Silverman Butts, 2011; Schumacher et al., 2001; Strobach, Frensch, Müller, \& Schubert, 2012a, 2012b; Strobach et al., 2008; Tombu \& Jolicoeur, 2004).

As illustrated in Figure $2 \mathrm{~A}$ and $2 \mathrm{~B}$, one specific realization of the optimized task coordination principle explaining the exclusive dualtask-practice advantage in the longer auditory task might be as following: The dual-task processing architecture includes (a) a within-task capacity limitation (i.e., bottleneck process) in the faster visual task (e.g., at a central response-selection stage) followed by (b) a switching operation, and (c) the within-task capacity limitation in the slower auditory task (Band \& van Nes, 2006; Lien, Schweickert, \& Proctor, 2003; Stelzel, Brandt, \& Schubert, 2009). The switching operation is theorized as activating and/or instantiating the rules that map Task 2 stimuli onto responses (Maquestiaux et al., 2004). It may be that the rules must be moved back into working memory or that the rules remaining in working memory throughout the task must be reestablished. After dual-task practice (see Figure 2A), in contrast to single-task practice (see Figure $2 \mathrm{~B})$, activation/instantiation processes are highly efficient due to task coordination skills, leading to a shortening of the switching operation: Participants might have learned to load task information faster or to load more information at a time into the working memory component. Since this shortened switching operation after the response-selection stage in the faster visual task is located before this stage in the slower auditory task, improved dual-task performance occurs in this latter task exclusively (see Strobach, Salminen, Karbach, \& Schubert, 2014, for a more detailed discussion).

Importantly, the conclusion of a shortened switching operation can be distinguished from a potential explanation of the previous findings from stage shortening within the component tasks. First, participants during dual-task and single-task practice received an identical number of stimulus contacts and thus component task experience, making a dual-task performance advantage after dual-task practice implausible from a methodological perspective (Liepelt, Strobach, et al., 2011; Strobach, Frensch, Soutschek, et al., 2012). Second, RTs in the single visual and auditory tasks were similar after single and dual-task practice. So, component task processing skills were equivalent, also making a single-task performance advantage after dual-task practice implausible from a results perspective. These arguments further distinguish the optimized task coordination principle from the task processing optimization principle. 

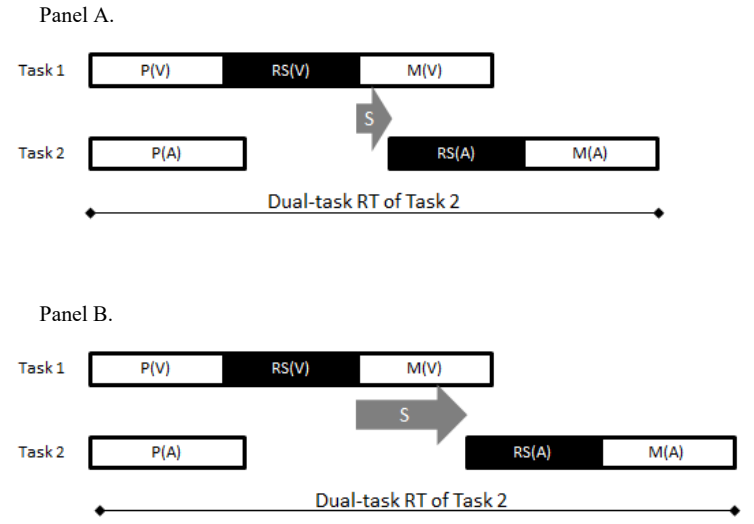

FIGURE 2.

Illustration of the hypothetical time relation of processing stages in Task 1 (e.g., a visual task) and Task 2 (e.g., an auditory tasks) as shorter and longer task, respectively, when presented in a dual-task situation with $\mathrm{SOA}=0 \mathrm{~ms} . \mathrm{P}(\mathrm{V})$ and $\mathrm{P}(\mathrm{A})$ indicate the perception stages; $\mathrm{RS}(\mathrm{V})$ and $\mathrm{RS}(\mathrm{A})$ indicate the central response-selection stages (including bottleneck characteristics); $M(V)$ and $M(A)$ indicate the motor stages; $\mathrm{S}$ indicates switching between component tasks after the completion of $\mathrm{RS}(\mathrm{V})$ and before the start of RS(A). Panel (A): Hypothetical time relation of dual-task processing at the end of dual-task practice including a short switch (i.e., optimized instantiation of information of two tasks) after the completion of RS(V) and before RS(A) leading to relatively short dual-task RTs in the auditory task. Panel B: Hypothetical time relation of dual-task processing at the end of single-task practice including no optimized switch after the completion of RS(V) and before RS(A) leading to relatively long dual-task RTs in the auditory task.

One important aspect is that the dual-task improvement after dual-task practice was evident not only in practiced dual tasks, but also in dual-task transfer situations that introduced changes in specific properties of either the visual or the auditory task (Liepelt, Strobach, et al., 2011; Strobach, Frensch, Müller, \& Schubert, 2015). These findings provide first evidence that acquired task coordination skills (potentially accounting for a better switching operation) are not tied to specific properties of the practiced component tasks but are transferable to different dual-task situations. This means that these findings preliminarily support the assumption of the transferability of acquired task coordination skills.

However, this transfer is limited to the practice context with combined visual and auditory tasks (Strobach, Frensch, Soutschek, et al., 2012): There is no evidence for a dual-task practice transfer (i.e., transfer of task coordination skills) to situations that introduce changes in specific properties of both the visual and the auditory task. In other words, transfer is only evident when one of the originally practiced tasks is present in the transfer context. Thus, investigating this visualauditory task combination provides no conclusive evidence that task coordination skills, acquired by dual-task in contrast to single-task practice, are transferable to unpracticed dual-task situations (i.e., dual tasks that share no component tasks with the practice situation). In consequence, there is only partial support for the optimized task coordination principle.

There are two contrasting hypotheses following from this set of assumptions on the optimized task coordination principle. First, while there is evidence for the acquisition of task coordination skills during dual-task in contrast to single-task practice, there is no conclusive evidence for the transferability of these skills to new situations. Hence, it can be disputed whether there is evidence that executive functions which impair dual-task performance at low levels of practice are associated with mechanisms explaining how the difficulty to perform two simultaneous tasks can be overcome and how dual-task costs are reduced at the end of practice.

Second, one explanation to secure the optimized task coordination principle in the context of the same-mechanism principle could be that the introduced dual-task practice situation was not variable enough to generate task coordination skills transferable to unpracticed dual tasks (Schmidt \& Bjork, 1992). The situation of Strobach, Frensch, Soutschek, et al. (2012) included identical visual and auditory component tasks, with only one SOA level ( $\mathrm{SOA}=0 \mathrm{~ms})$ across the entire practice phase. In line with this assumption, there exist a number of studies testing the acquisition and transferability of task coordination skills with real-world dual-task practice. These tasks are supposed to provide a natural variability during dual-task practice.

For instance, the case of persons with experience in simultaneous interpreting, in contrast to persons with consecutive interpreting experience, might represent a contrast between persons with dual-task practice with a natural variability and no such practice, respectively (Becker, Schubert, Strobach, Gallinat, \& Kühn, 2016; Strobach, Becker, Schubert, \& Kühn, 2015). In fact, simultaneous interpreting is characterized by simultaneous listening and speaking (i.e., dual tasks) of a (theoretically) infinite amount of language material (i.e., sentences, phrases, etc.). In contrast to that, consecutive interpreting requires multiple task coordination to a lesser degree (rather single tasks with non-simultaneous but sequential alternations between listening and speaking). As a consequence, it can be hypothesized that dual-task performance is better in simultaneous interpreters in contrast to consecutive interpreters in a PRP situation combining an auditory Task 1 and a visual Task 2 that are not related to tasks conducted during interpreting (neither simultaneous nor consecutive). Because of the unrelated component tasks in the interpreting and PRP context, improved dual-task performance in simultaneous in contrast to consecutive interpreters should not be associated with optimizations in component task processing. However, this improvement should be related to the practice-related plasticity and transferability of task coordination skills resulting from simultaneous interpreting. In fact, RTs in Task 1 and Task 2 were reduced in simultaneous interpreters in contrast to consecutive interpreters. So, data of the PRP dual-task situation is consistent with the assumption of transferable task coordination skills and thus provide support for the optimized task coordination principle. Importantly, this support for the optimized task coordination processes can be distinguished from stage shortening within the component 
tasks (i.e., task processing optimization principle). In fact, reduced RTs for simultaneous interpreters in Task 1 and Task 2 were exclusively observed under dual-task conditions but not in single tasks. So, component task processing skills were equivalent in making a single-task performance advantage in simultaneous interpreters implausible from a results perspective.

Similar conclusions can be drawn from investigations of persons with extensive experience in playing video games. In fact, when persons are experienced in action video games (requiring multiple simultaneous tasks and actions which classify as dual tasking) and are tested in a PRP dual task similar to simultaneous and consecutive interpreters (see above), their RTs are reduced in contrast to RTs of persons with no such experience (Strobach, Frensch, \& Schubert, 2012; see also Chiappe, Conger, Liao, Caldwell, \& Vu, 2013); single-task RTs do not change between groups. Again, PRP component tasks are not related to the context of experience (i.e., video games). Hence, action video gamers might have optimized task coordination skills that were acquired from a variable real-world task and were demonstrated in a lab-based situation with simple component tasks. This set of conclusions is consistent with the assumption of transferable task coordination skills and the optimized task coordination principle. Besides that, this assumption reveals the validity of the same-mechanism principle, assuming that difficulties to perform two simultaneous tasks at the beginning of practice due to executive functioning might be overcome by the acquisition of task coordination skills.

\section{SUMMARY}

To identify mechanisms leading to a practice-related improvement in dual tasks with simple tasks, the present review assessed the validity of the same-mechanism principle: Sources that impair dual-task performance at low levels of practice are associated with mechanisms that explain how the difficulty to perform two simultaneous tasks can be overcome and how dual-task costs are reduced at the end of practice. The following processes provide sources for the occurrence of dualtask costs at low practice levels: (a) processes within the component tasks as well as (b) processes independent of these tasks and thus outside of them. These processes are associated with the optimized task processing principle (i.e., shortening and automatization of capacitylimited processes within the component tasks), the optimized attention allocation principle (i.e., improved allocation of limited attention resources to two tasks and the acquisition of related skills outside of the component tasks), as well as the optimized task coordination principle (i.e., improved control and regulation of two tasks and the acquisition of related skills outside of the component tasks). The present literature review showed that there is substantial evidence for the optimized task processing principle (i.e., central bottleneck stage shortening and task automatization), substantial evidence for the optimized attention allocation principle, as well as some evidence for the optimized task coordination principle. Exclusively in the context of the motor stage bottleneck, the same-mechanism principle seems invalid since there is no evidence that this source of dual-task costs at low practice levels is associated with mechanisms that contribute to the improvement of dual-task performance during practice.

What are potential conclusions from this research to inform not only practice-related dual-task improvements with simple component tasks but also improvements in complex kinds of mental activities? Of course, real-world activities are not exclusively composed of simple tasks and discrete stimulus-response events. Similarly, illustrations of processing architectures in Figures 1 and 2 are not illustrations of the complex mental apparatus. However, these illustrations, as well as conclusions from research on simple tasks, demonstrate the time course of basic mental processes (i.e., perception, decision) and their unfolding in time when certain demands are imposed on the human information processing system. In this review, these demonstrations were made under practiced dual-task conditions. From this perspective, research with simple component tasks has the potential to inform human behavior outside the laboratory.

\section{AUTHOR NOTES}

The present research was supported by grants of the German Research Foundation (Deutsche Forschungsgemeinschaft, Fr 1388/8-1, Schu 1397/ 3-1, 3-2, Schu 1397/5-1, 5-2, STR 1223/1-1). We wish to thank Franziska Orscheschek for proofreading the manuscript versions and three anonymous reviewers for their helpful comments on a previous version of this manuscript.

\section{REFERENCES}

Ahissar, M., Laiwand, R., \& Hochstein, S. (2001). Attentional demands following perceptual skill training. Psychological Science, 12, 56-62. doi: 10.1111/1467-9280.00310 [wW

Anderson, J. R., Taatgen, N. A., \& Byrne, M. D. (2005). Learning to achieve perfect time sharing: Architectural implications of Hazeltine, Teague, \& Ivry (2002). Journal of Experimental Psychology: Human Perception and Performance, 31, 749-761. doi: 10.1037/0096-1523.31.4.749 wWw

Anguera, J. A., Boccanfuso, J., Rintoul, J. L., Al-Hashimi, O., Faraji, F., Janowich, J., . . Gazzaley, A. (2013). Video game training enhances cognitive control in older adults. Nature, 501, 97-101. doi: 10.1038/nature12486 WWW

Band, G. P. H., \& van Nes, F. T. (2006). Reconfiguration and the bottleneck: Does task switching affect the refractory-period effect? European Journal of Cognitive Psychology, 18, 593-623. doi: 10.1080/09541440500423244

Becker, M., Schubert, T., Strobach, T., Gallinat, J., \& Kühn, S. (2016). Simultaneous interpreters vs. professional multilingual controls: Group differences in cognitive control as well as brain structure and function. Neurolmage, 134, 250-260. doi:

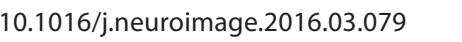

Bherer, L., Kramer, A. F., Peterson, M. S., Colcombe, S., Erickson, K., $\&$ Becic, E. (2005). Training effects on dual-task performance: Are there age-related differences in plasticity of attentional control? Psychology and Aging, 20, 695-709. doi: 10.1037/08827974.20.4.695 |WWW 
Bherer, L., Kramer, A. F., Peterson, M. S., Colcombe, S., Erickson, K., \& Becic, E. (2006). Testing the limits of cognitive plasticity in older adults: Application to attentional control. Acta Psychologica, 123, 261-278. doi: 10.1016/j.actpsy.2006.01.005 WWW

Bherer, L., Kramer, A. F., Peterson, M. S., Colcombe, S., Erickson, K., \& Becic, E. (2008). Transfer effects in task-set cost and dualtask cost after dual-task training in older and younger adults: Further evidence for cognitive plasticity in attentional control in late adulthood. Experimental Aging Research, 34, 188-209. doi: 10.1080/03610730802070068 WWW

Bier, B., de Boysson, C., \& Belleville, S. (2014). Identifying training modalities to improve multitasking in older adults. Age, 36,

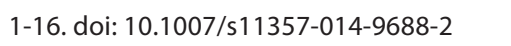

Bratzke, D., Rolke, B., \& Ulrich, R. (2009). The source of executionrelated dual-task interference: Motor bottleneck or response monitoring? Journal of Experimental Psychology: Human Perception and Performance, 35, 1413-1426. doi: 10.1037/ a0015874 WWW

Chiappe, D., Conger, M., Liao, J., Caldwell, J., \& Vu, K. L. (2013). Improving multi-tasking ability through action videogames. Applied Ergonomics, 44, 278-284. doi: 10.1016/j.

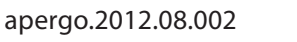

Damos, D. L., \& Wickens, C. D. (1980). The identification and transfer of timesharing skills. Acta Psychologica, 46, 15-39. doi: 10.1016/0001-6918(80)90057-8

De Jong, R. (1993). Multiple bottlenecks in overlapping task performance. Journal of Experimental Psychology: Human Perception and Performance, 19, 965-980. doi: 10.1037/00961523.19.5.965 WWW

De Jong, R. (1995). The role of preparation in overlapping-task performance. Quarterly Journal of Experimental Psychology, 48A, 2-25. doi: 10.1080/14640749508401372 |wWw

Dux, P. E., Tombu, M. N., Harrison, S., Rogers, B. P., Tong, F., \& Marois, R. (2009). Training improves multitasking performance by increasing the speed of information processing in human prefrontal cortex. Neuron, 63, 127-138. doi: 10.1016/j.

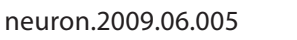

Ellenbogen, R., \& Meiran, N. (2008). Working memory involvement in dual-task performance: Evidence from the backward compatibility effect. Memory \& Cognition, 36, 968-978. doi: 10.3758/MC.36.5.968 $\overline{\mathrm{WWW}}$

Fischer, R., \& Plessow, F. (2015). Efficient multitasking: Parallel versus serial processing of multiple tasks. Frontiers in Psychology, 6. doi: 10.3389/fpsyg.2015.01366 |WWW

Gagnon, L. G., \& Belleville, S. (2012). Training of attentional control in mild cognitive impairment with executive deficits: Results from a double-blind randomised controlled study. Neuropsychological Rehabilitation, 22, 809-835. doi:

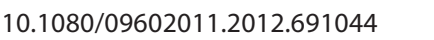

Garner, K. G., \& Dux, P. E. (2015). Training conquers multitasking costs by dividing task representations in the frontoparietalsubcortical system. Proceedings of the National Academy of
Sciences of the United States of America, 112, 14372-14377. doi: 10.1073/pnas.1511423112 WWW

Garner, K. G., Lynch, C. R., \& Dux, P. E. (2016). Transfer of training benefits requires rules we cannot see (or hear). Journal of Experimental Psychology: Human Perception and Performance,

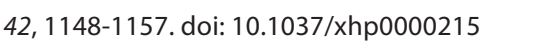

Garner, K. G., Tombu, M. N., \& Dux, P. E. (2014). The influence of training on the attentional blink and psychological refractory period. Attention, Perception, \& Psychophysics, 76, 979-999. doi: 10.3758/s13414-014-0638-y

Gopher, D., Brickner, M., \& Navon, D. (1982). Different difficulty manipulations interact differently with task emphasis: Evidence for multiple resources. Journal of Experimental Psychology: Human Perception and Performance, 8, 146-157. doi: 10.1037/0096-1523.8.1.146 WwW

Göthe, K., Oberauer, K., \& Kliegl, R. (2016). Eliminating dualtask costs by minimizing crosstalk between tasks: The role of modality and feature pairings. Cognition, 150, 92-108. doi: 10.1016/j.cognition.2016.02.003 WW

Hampson, P. J. (1989). Aspects of attention and cognitive science. The Irish Journal of Psychology, 10, 261-275. doi: 10.1080/03033910.1989.10557746

Hartley, A. A., Maquestiaux, F., \& Silverman Butts, N. S. (2011). A demonstration of dual-task performance without interference in some older adults. Psychology and Aging, 26, 181-187. doi: 10.1037/a0021497 |

Hazeltine, E., Teague, D., \& Ivry, R. B. (2002). Simultaneous dualtask performance reveals parallel response selection after practice. Journal of Experimental Psychology: Human Perception and Performance, 28, 527-545. doi: 10.1037/0096-1523.28.3.527 WWW

Hirst, W., Spelke, E. S., Reaves, C. C., Caharack, G., \& Neisser, U. (1980). Dividing attention without alteration or automaticity. Journal of Experimental Psychology: General, 109, 98-117. doi: 10.1037/0096-3445.109.1.98

Hommel, B. (1998). Automatic stimulus-response translation in dual-task performance. Journal of Experimental Psychology: Human Perception and Performance, 24, 1368-1384. doi: 10.1037/0096-1523.24.5.1368 "WW

Hommel, B., \& Eglau, B. (2002). Control of stimulus-response translation in dual-task performance. Psychological Research, 66, 260-273. doi: 10.1007/s00426-002-0100-y

Janczyk, M., Pfister, R., Hommel, B., \& Kunde, W. (2014). Who is talking in backward crosstalk? Disentangling response-from goal-conflict in dual-task performance. Cognition, 132, 30-43. doi: 10.1016/j.cognition.2014.03.001 $\overline{\underline{W W}}$

Jiang, Y., Saxe, R., \& Kanwisher, N. (2004). Functional magnetic resonance imaging provides new constraints on theories of the psychological refractory period. Psychological Science, 15, 390-396. doi: 10.1111/j.0956-7976.2004.00690.x |WWW|

Kahneman, D. (1973). Attention and effort. Englewood Cliffs, NJ: Prentice Hall. 
Kamienkowski, J. E., Pashler, H., Sigman, M., \& Dehaene, S. (2011). Effects of practice on task architecture: Combined evidence from interference experiments and random-walk models of decision making. Cognition, 119, 81-95. doi: 10.1016/j. cognition.2010.12.010 WWW

Korteling, J. E. (1991). Effects of skill integration and perceptual competition on age-related differences in dualtask performance. Human Factors: The Journal of the Human Factors and Ergonomics Society, 33, 35-44. doi: $10.1177 / 001872089103300103$ WWW

Kramer, A. F., Larish, J. F., \& Strayer, D. L. (1995). Training for attentional control in dual task settings: A comparison of young and old adults. Journal of Experimental Psychology: Applied, 1, 50-76. doi: 10.1037/1076-898X.1.1.50

Kramer, A.F., Larish, J., Weber, T., \& Bardell, L. (1999). Training for executive control: Task coordination strategies and aging. In D. Gopher \& A. Koriat (Eds.), Attention and performance XVII (pp. 617-652). Cambridge, MA. MIT Press.

Lee, H., Voss, M. W., Prakash, R. S., Boot, W. R., Vo, L. T., Basak, C., ... Kramer, A. F. (2012). Videogame training strategy-induced change in brain function during a complex visuomotor task. Behavioural Brain Research, 232, 348-357. doi: 10.1016/j.

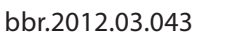

Levy, J., \& Pashler, H. (1995). Does perceptual analysis continue during selection and production of a speeded response? Acta Psychologica, 90, 245-260. doi: 10.1016/0001-6918(95)00025-P WWW

Lien, M.-C., Schweickert, R., \& Proctor, R. W. (2003). Task switching and response correspondence in the psychological refractory period paradigm. Journal of Experimental Psychology: Human Perception and Performance, 29, 692-712. doi: 10.1037/00961523.29.3.692 WWW

Liepelt, R., Fischer, R., Frensch, P. A., \& Schubert, T. (2011). Practice-related reduction of dual-task costs under conditions of a manual-pedal response combination. Journal of Cognitive Psychology, 23, 29-44. doi: 10.1080/20445911.2011.448025

Liepelt, R., Strobach, T., Frensch, P. A., \& Schubert, T. (2011). Improved inter-task coordination after extensive dual-task practice. Quarterly Journal of Experimental Psychology, 64, 1251-1272. doi: 10.1080/17470218.2010.543284|wW|

Logan, G. D. (1988). Toward an instance theory of automatization. Psychological Review, 95, 492-527. doi: 10.1037/0033-295X.95.4.492

Logan, G. D., \& Gordon, R. (2001). Executive control of visual attention in dual-task situations. Psychological Review, 108, 393-

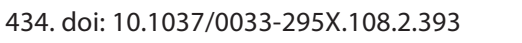

Luria, R., \& Meiran, N. (2003). Online order control in the psychological refractory period paradigm. Journal of Experimental Psychology: Human Perception and Performance, 29, 556-574. doi: 10.1037/0096-1523.29.3.556 [WWW

Lussier, M., Gagnon, C., \& Bherer, L. (2012). An investigation of response and stimulus modality transfer effects after dual-task training in younger and. Frontiers in Human Neuroscience, 6. doi: 10.3389/fnhum.2012.00129 $\underline{\underline{W W}}$

Maquestiaux, F., Didierjean, A., Ruthruff, E., Chauvel, G., \& Hartley, A. (2013). Lost ability to automatize task performance in old age. Psychonomic Bulletin \& Review, 20, 1206-1212. doi: 10.3758/s13423-013-0438-8 WwW

Maquestiaux, F., Hartley, A. A., \& Bertsch, J. (2004). Can practice overcome age-related differences in the psychological refractory period effect? Psychology and Aging, 19, 649-667. doi: 10.1037/0882-7974.19.4.649 $\overline{\mathrm{WWW}}$

Maquestiaux, F., Laguë-Beauvais, M., Ruthruff, E., \& Bherer, L. (2008). Bypassing the central bottleneck after single-task practice in the psychological refractory period paradigm: Evidence for task automatization and greedy resource recruitment. Memory \& Cognition, 36, 1262-1282. doi: 10.3758/MC.36.7.1262 WWW

Maquestiaux, F., Laguë-Beauvais, M., Ruthruff, E., Hartley, A. A., \& Bherer, L. (2010). Learning to bypass the central bottleneck: Declining automaticity with advancing age. Psychology and Aging, 25, 177-192. doi: 10.1037/a0017122 |WWw

Masaki, H., Takasawa, N., \& Yamazaki, K. (2000). An electrophysiological study of the locus of the interference effect in a stimulus response compatibility paradigm. Psychophysiology, 37, 464-472. doi: 10.1111/1469-8986.3740464 |WWW|

McCann, R. S., \& Johnston, J. C. (1992). Locus of the single-channel bottleneck in dual-task interference. Journal of Experimental Psychology: Human Perception and Performance, 18, 471-484. doi: 10.1037/0096-1523.18.2.471

Meyer, D. E., \& Kieras, D. E. (1997). A computational theory of executive cognitive processes and multiple-task performance: Part 2. Accounts of psychological refractory-period phenomena. Psychological Review, 104, 749-791. doi: 10.1037//0033295X.104.4.749

Miller, J., Ulrich, R., \& Rolke, B. (2009). On the optimality of serial and parallel processing in the psychological refractory period paradigm: Effects of the distribution of stimulus onset asynchronies. Cognitive Psychology, 58, 273-310. doi: 10.1016/j. cogpsych.2006.08.003

Miyake, A., \& Friedman, N. P. (2012). The nature and organization of individual differences in executive functions four general conclusions. Current Directions in Psychological Science, 21, 8-14. doi: 10.1177/0963721411429458 WWW

Miyake, A., Friedman, N. P., Emerson, M. J., Witzki, A. H., Howerter, A., \& Wager, T. D. (2000). The unity and diversity of executive functions and their contributions to complex "frontal lobe" tasks: A latent variable analysis. Cognitive Psychology, 41, 49100. doi: 10.1006/cogp.1999.0734 WWW

Navon, D., \& Gopher, D. (1979). On the economy of the humanprocessing system. Psychological Review, 86, 214-255. doi: 10.1037/0033-295X.86.3.214

Navon, D., \& Miller, J. (2002). Queuing or sharing? A critical evaluation of the single-bottleneck notion. Cognitive Psychology, 44,

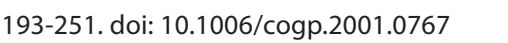


Norman, D. A., \& Bobrow, D. G. (1975). On data-limited and resource-limited processes. Cognitive Psychology, 7, 44-64. doi: 10.1016/0010-0285(75)90004-3

Oberauer, K., \& Kliegl, R. (2004). Simultaneous cognitive operations in working memory after dual-task practice. Journal of Experimental Psychology: Human Perception and Performance, 30, 689-707. doi: 10.1037/0096-1523.30.4.689 |WW

Pashler, H. (1984). Processing stages in overlapping tasks: Evidence for a central bottleneck. Journal of Experimental Psychology: Human Perception and Performance, 10, 358-377. doi: 10.1037/0096-1523.10.3.358 WWW

Pashler, H. (1994a). Dual-task interference in simple tasks: Data and theory. Psychological Bulletin, 116, 220-244. doi: 10.1037/0033-2909.116.2.220 $\underline{\underline{W W} \mid}$

Pashler, H. (1994b). Graded capacity-sharing in dual-task interference? Journal of Experimental Psychology: Human Perception and Performance, 20, 330-342. doi: 10.1037/0096-1523.20.2.330 WWW

Pashler, H., \& Baylis, G. (1991). Procedural learning: I. Locus of practice effects in speeded choice tasks. Journal of Experimental Psychology: Learning, Memory and Cognition, 17, 20-32. doi: 10.1037/0278-7393.17.1.20

Pashler, H., \& Johnston, J. C. (1989). Chronometric evidence for central postponement in temporally overlapping tasks. The Quarterly Journal of Experimental Psychology, 41, 19-45. doi: 10.1080/14640748908402351

Pashler, H., \& Johnston, J. C. (1998). Attentional limitations in dual-task performance. In H. Pashler (Ed.), Attention (pp. 155189). Hove, England: Psychology Press/Erlbaum.

Ruthruff, E., Johnston, J. C., \& Van Selst, M. V. (2001). Why practice reduces dual-task interference. Journal of Experimental Psychology: Human Perception and Performance, 27, 3-21. doi: 10.1037/0096-1523.27.1.3 $\overline{\mathrm{WWW}}$

Ruthruff, E., Johnston, J. C., Van Selst, M. V., Whitsell, S., \& Remington, R. (2003). Vanishing dual-task interference after practice: Has the bottleneck been eliminated or is it merely latent? Journal of Experimental Psychology: Human Perception and Performance, 29, 280-289. doi: 10.1037/0096-1523.29.2.280 WWW

Ruthruff, E., Van Selst, M., Johnston, J. C., \& Remington, R. W. (2006). How does practice reduce dual-task interference: Integration, automatization, or simply stage-shortening? Psychological Research, 70, 125-142. doi: 10.1007/s00426-004-0192-7 WWW

Sangals, J., Wilwer, M., \& Sommer, W. (2007). Localising practice effects in dual-taskperformance.Quarterly Journal of Experimental Psychology, 60, 860-876. doi: 10.1080/17470210600822720 WWW

Schmidt, A., \& Bjork, R. A. (1992). New conceptualizations of practice: Common principles in three paradigms suggest new concepts from training. Psychological Science, 3, 207-217. doi: 10.1111/j.1467-9280.1992.tb00029.x
Schneider, W., \& Shiffrin, R. M. (1977). Controlled and automatic human information processing: I. Detection, search, and attention. Psychological Review, 84, 1-66. doi: 10.1037/0033-295X.84.1.1

Schubert, T. (1999). Processing differences between simple and choice reaction affect bottleneck localization in overlapping tasks. Journal of Experimental Psychology: Human Perception and Performance, 25, 408-425. doi: 10.1037/0096-1523.25.2.408

Schubert, T. (2008). The central attentional limitation and execu-

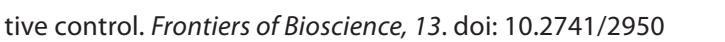

Schubert, T., Fischer, R., \& Stelzel, C. (2008). Response activation in overlapping tasks and the response selection bottleneck. Journal of Experimental Psychology: Human Perception and Performance, 34, 376-397. doi: 10.1037/0096-1523.34.2.376 WWW

Schubert, T., \& Szameitat, A. J. (2003). Functional neuroanatomy of interference in overlapping dual tasks: An fMRI study. Cognitive Brain Research, 17, 733-746. doi: 10.1016/S09266410(03)00198-8 $\underline{\underline{W W}}$

Schumacher, E. H., Seymour, T. L., Glass, J. M., Fencsik, D. E., Lauber, E. J., Kieras, D. E., \& Meyer, D. E. (2001). Virtually perfect time sharing in dual-task performance: Uncorking the central cognitive bottleneck. Psychological Science, 12(2), 101-108. doi:

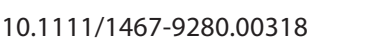

Sigman, M., \& Dehaene, S. (2006). Dynamics of the central bottleneck: Dual-task and task uncertainty. PLOS: Biology, 4. doi: 10.1371/journal.pbio.0040220 $\underline{\underline{W W}}$

Sommer, W., Leuthold, H., \& Schubert, T. (2001). Multiple bottlenecks in information processing? An electrophysiological examination. Psychonomic Bulletin \& Review, 8, 81-88. doi:10.3758/ BF03196142 |WW|

Stelzel, C., Brandt, S. A., \& Schubert, T. (2009). Neural mechanisms of concurrent stimulus processing in dual tasks. Neurolmage, 48, 237-248. doi: 10.1016/j.neuroimage.2009.06.064 WWW

Strobach, T., Becker, M., Schubert, T., \& Kühn, S. (2015). Better dual-task processing in simultaneous interpreters. Frontiers in Psychology, 6. doi: 10.3389/fpsyg.2015.01590 WWW

Strobach, T., Frensch, P. A., Müller, H., \& Schubert, T. (2012a). Age- and practice-related influences on dual-task costs and compensation mechanisms under optimal conditions for dual-task performance. Aging, Neuropsychology, \& Cognition, 19, 222-247. doi: 10.1080/13825585.2011.630973 WWW

Strobach, T., Frensch, P. A., Müller, H., \& Schubert, T. (2012b). Testing the limits of optimizing dual-task performance in younger and older adults. Frontiers in Human Neuroscience, 6. doi: 10.3389/fnhum.2012.00039 |WWW

Strobach, T., Frensch, P., Müller, H., \& Schubert, T. (2015). Evidence for the acquisition of dual-task coordination skills in older adults. Acta Psychologica, 160, 104-116. doi: 10.1016/j.

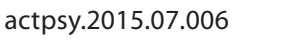

Strobach, T., Frensch, P. A., \& Schubert, T. (2008). The temporal stability of skilled dual-task performance. In H. D. Zimmer, C. 
Frings, A. Mecklinger, B. Opitz, M. Pospeschill, \& D. Wentura (Eds.), Cognitive Science 2007. Proceedings of the 8th Annual Conference of the Cognitive Science Society of Germany (pp. 1-13). Saarbrücken, Germany.

Strobach, T., Frensch, P. A., \& Schubert, T. (2012). Video game practice optimizes executive control skills in dual-task and task switching situations. Acta Psychologica, 140(1), 13-24. doi: 10.1016/j.actpsy.2012.02.001 WWW

Strobach, T., Frensch, P. A., Soutschek, A., \& Schubert, T. (2012). Investigation on the improvement and transfer of dual-task coordination skills. Psychological Research, 76, 794-811. doi: 10.1007/s00426-011-0381-0 WWW

Strobach, T., Gerstorf, D., Maquestiaux, F., \& Schubert, T. (2015). Does initial performance variability predict dual-task optimization with practice in tounger and older adults? Experimental Aging Research, 41, 57-88. doi: 10.1080/0361073X.2015.978210 WWW

Strobach, T., Liepelt, R., Pashler, H., Frensch, P. A., \& Schubert, T. (2013). Effects of extensive dual-task practice on processing stages in simultaneous choice tasks. Attention, Perception, \& Psychophysics, 75, 900-920. doi: 10.3758/s13414-013-0451-z WWW

Strobach, T., Salminen, T., Karbach, J., \& Schubert, T. (2014). Practice-related optimization and transfer of executive functions: A general review and a specific realization of their mechanisms in dual tasks. Psychological Research, 78, 836-851. doi: 10.1007/s00426-014-0563-7 WwW

Strobach, T., Schubert, T., Pashler, H., \& Rickard, T. (2014). The specificity of learned parallelism in dual-memory retrieval. Memory \& Cognition, 42, 552-569. doi: 10.3758/s13421-013-

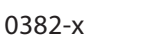

Strobach, T., Schütz, A., \& Schubert, T. (2015). On the importance of task 1 and error performance measures in PRP dual-task studies. Frontiers in Psychology, 6. doi: 10.3389/fpsyg.2015.00403 $\overline{\mathrm{WWW}}$

Szameitat, A. J., Lepsien, J., von Cramon, D. Y., Sterr, A., \& Schubert, T. (2006). Task-order coordination in dual-task performance and the lateral prefrontal cortex: An event-related fMRI study. Psychological Research, 70, 541-552. doi: 10.1007/s00426-0050015-5 WWW

Telford, C. W. (1931). The refractory phase of voluntary and associative responses. Journal of Experimental Psychology, 14, 1-36. doi: 10.1037/h0073262

Thomson, S. J., Danis, L. K., \& Watter, S. (2015). PRP training shows task 1 response selection is the locus of the backward response compatibility effect. Psychonomic Bulletin \& Review, 22, 212218. doi: 10.3758/s13423-014-0660-z $\underline{\text { WWW }}$

Tombu, M., \& Jolicœur, P. (2003). A central capacity sharing model of dual-task performance. Journal of Experimental Psychology: Human Perception and Performance, 29, 3-18. doi: 10.1037/0096-1523.29.1.3 $\underline{\text { WWW }}$

Tombu, M., \& Jolicoeur, P. (2004). Virtually no evidence for virtually perfect time-sharing. Journal of Experimental Psychology: Human Perception and Performance, 30, 795-810. doi: 10.1037/0096-1523.30.5.795 wWw

Van Selst, M., Ruthruff, E., \& Johnston, J. C. (1999). Can practice eliminate the Psychological Refractory Period Effect? Journal of Experimental Psychology: Human Perception and Performance, 25, 1268-1283. doi: 10.1037/0096-1523.25.5.1268 www

Voss, M. W., Prakash, R. S., Erickson, K. I., Boot, W. R., Basak, C., Neider, M. B., ... Kramer, A. F. (2012). Effects of training strategies implemented in a complex videogame on functional connectivity of attentional networks. Neurolmage, 59, 138-148. doi: 10.1016/j.neuroimage.2011.03.052 [WWW

Watter, S., \& Logan, G. D. (2006). Parallel response selection processes in dual-task situations. Perception \& Psychophysics, 68, 254-277. doi: 10.3758/BF03193674 WW

Welford, A. T. (1952). The psychological refractory period and the timing of high speed performance: A review and a theory. British Journal of Psychology, 43, 2-19. doi: 10.1111/j.20448295.1952.tb00322.x

Wickens, C. D. (2008). Multiple resources and mental workload. Human Factors: The Journal of the Human Factors and Ergonomics Society, 50, 449-455. doi: 10.1518/001872008X288394 |wWW

RECEIVED 26.07.2016 | ACCEPTED 01.11.2016 